\title{
TEM and Atom Probe Tomography Characterization of High-TMR MgO-Based Magnetic Tunnel Junctions
}

\author{
P.M. Rice, ${ }^{*}$ S-H. Yang, * S.S.P. Parkin, ${ }^{*}$ E. A. Marquis, ${ }^{* *}$ T.J. Prosa, ${ }^{* * *}$ and D.J. Larson*** \\ * IBM Almaden Research Center, 650 Harry Rd., San Jose, CA 95120 \\ ** Department of Materials, University of Oxford, Parks Road, Oxford, OX1 3PH, UK \\ *** Imago Scientific Instruments Corp., 5500 Nobel Drive, Madison, WI 53719
}

Magnetic tunnel junctions (MTJ) have significant technological value as non-volatile magnetic random-access memory (MRAM) and as magnetic sensors like those used in hard-disk drives for computers. MgO-based MTJs are particularly useful because of their high tunneling magnetoresistance (TMR) values at room temperature [1].

The MRAM structure consists of four main layers, Fig. 1. First, an anti-ferromagnetic layer, which in the samples discussed here is IrMn (22/78 atomic fraction), a disordered fcc material used because it is anti-ferromagnetic in both the as-deposited and annealed state. Second, is a ferromagnetic layer, in this case $\mathrm{CoFe}$ (70/30). The magnetic moment of this bcc structured material is pinned by the uncompensated spins at the top surface of the anti-ferromagnetic layer. The next layer is the tunneling barrier layer, in this case $\mathrm{MgO}$, an ordered $\mathrm{B} 1$ structure material with a band structure that matches well to that of $\mathrm{CoFe}$. The last layer is another ferromagnetic layer, in this case a combination of $\mathrm{CoFe}(70 / 30) / \mathrm{CoFeB}(49 / 21 / 30)$. This layer's magnetic moment is "free" to respond to external magnetic fields. The resistance of this structure differs significantly depending on whether the free layer's magnetic moment is aligned parallel (low resistance) or anti-parallel (high resistance) to the moment in the pinned layer. The magnitude of the difference between these values divided by the low resistance value is known as tunneling magneto-resistance (TMR). The values for TMR change as a function of annealing temperature, starting at around $100 \%$ in the as-deposited state and peaking at around $350 \%$ after an anneal of $400^{\circ} \mathrm{C}$ for 30 minutes [1]. For anneals above this temperature the TMR values begin to decline.

We have characterized this high-TMR MTJ structure in both the as-deposited and annealed states using analytical TEM and atom probe tomography (APT). The TEM was conducted at the IBM Almaden Research Center using a JEOL 2010F equipped with a Gatan Enfina EELS spectrometer. The atom probe tips were prepared by focused ion beam lift-out methods [2], and the atom probe data were acquired in an Imago LEAP 3000 XHR in the Materials Department at the University of Oxford.

Fig. 2 shows a summary of the results for the structure in the annealed state. An APT reconstruction is shown in Fig. 2a, an HREM image in Fig. 2b, and an EELS profile in Fig. 2c. All atom species in Fig. 2a except for Mg are color-coded to match the EELS data in Fig. 2c. Both the atom probe and the EELS data show that, in the annealed state, Mn atoms have diffused from the IrMn layer through the pinned $\mathrm{CoFe}$ layer and segregated to the pinned layer/oxide interface, with a small portion of $\mathrm{Mn}$ atoms left in the CoFe layer between the $\mathrm{MgO}$ barrier and the IrMn layer. Compositional analysis of a $4 \times 4 \times 4 n m$ volume from the APT reconstruction of the CoFe pinned layer in Fig. 2a yields a concentration of $1.6 \% \pm 0.3 \%$ at. $\mathrm{Mn}$ in the CoFe pinned layer. Comparisons of the atom probe data and the EELS data will be discussed in detail.

[1] S.S.P. Parkin et al., Nature Materials. 3 (12) (2004) 862.

[2] K. Thompson et al., Ultramicroscopy 107(2-3) (2006) 131.

[3] Authors would like to acknowledge the work of L.E. Krupp for TEM sample preparation. 


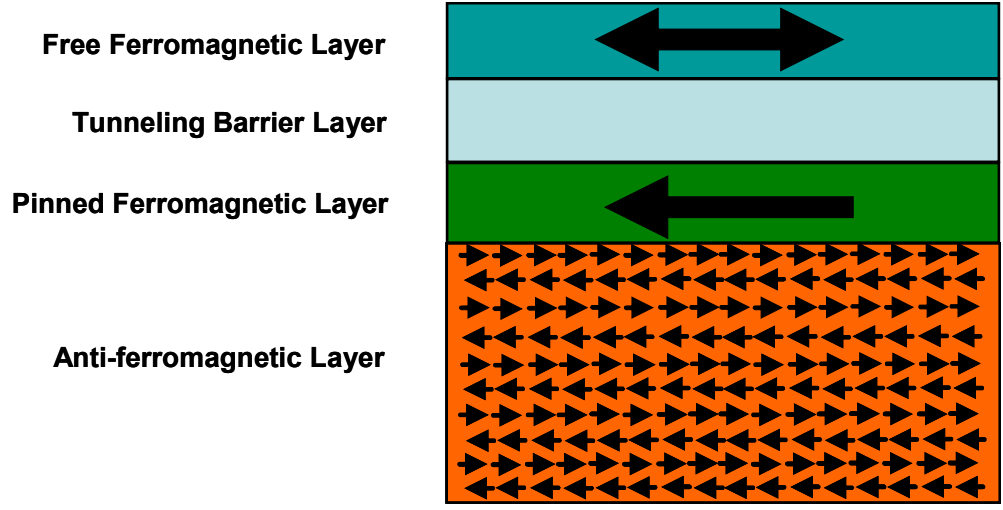

FIG. 1. MTJ structure used in magnetic memory and sensor technology. Arrows represent magnetic flux.

a)

b)
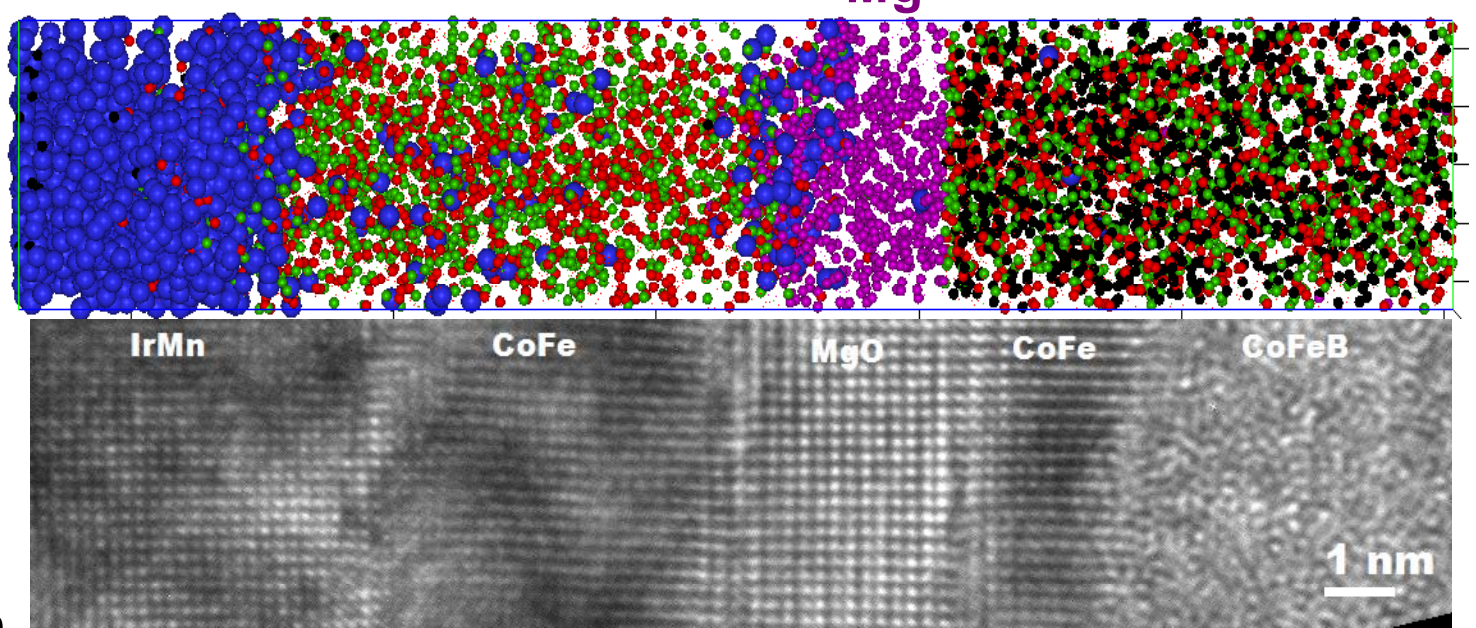

c)

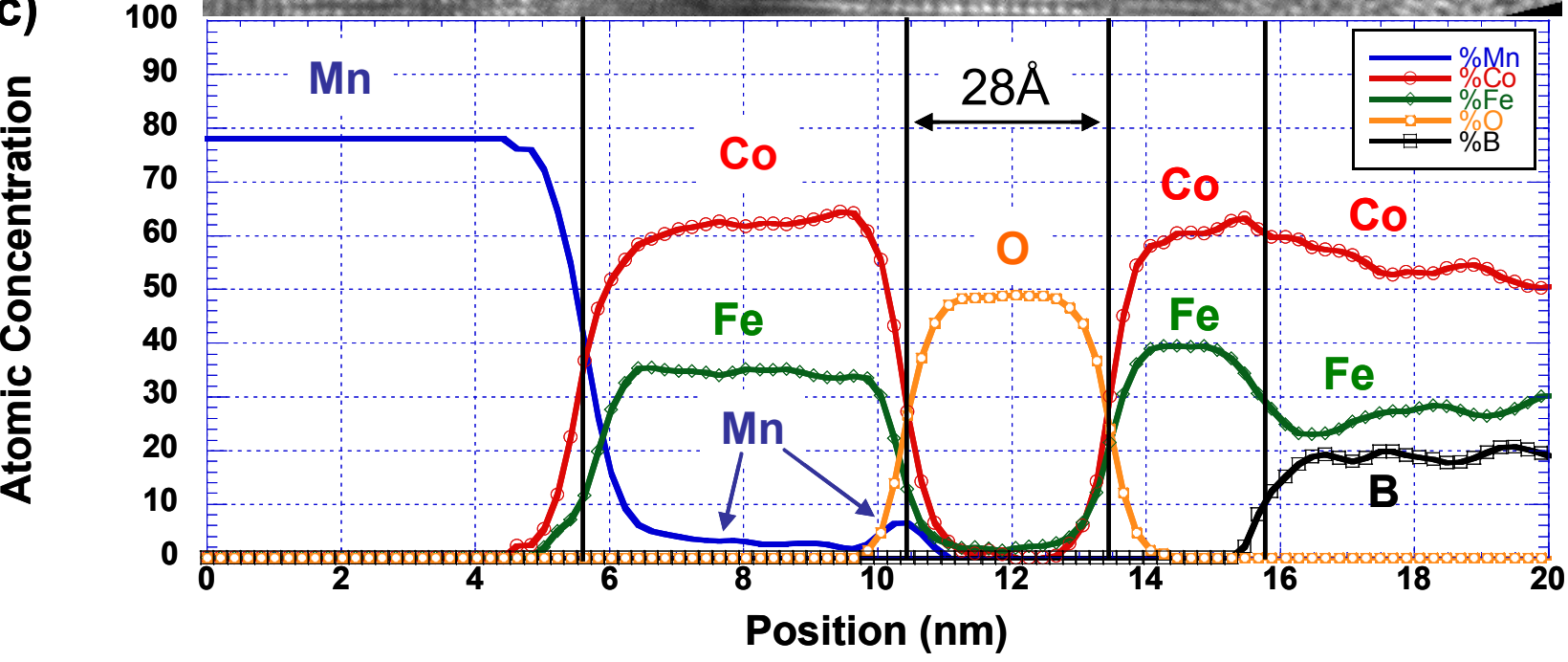

FIG. 2. MTJ structure in the annealed state. (a) atom probe tomography reconstruction with elements the same color as the EELS profile (except for Mg which was not measured in EELS), (b) High resolution TEM image showing the epitaxy between the layers, and (c) EELS profile acquired with $2 \AA$ steps across the layers showing Mn has diffused through the CoFe pinned layer and has segregated to the bottom oxide interface. 